\title{
Antineutrophil cytoplasm antibodies directed against myeloperoxidase augment leukocyte-microvascular interactions in vivo
}

\author{
Mark A. Little, C. Lucy Smyth, Rashmi Yadav, Lyn Ambrose, H. Terence Cook, Sussan Nourshargh, and Charles D. Pusey
}

Systemic small vessel vasculitis is associated with antineutrophil cytoplasm antibodies (ANCAs). While there is mounting in vitro evidence to suggest that ANCAs are capable of enhancing leukocyte-endothelial interactions, no in vivo evidence for this has been provided. In this study a novel rat model of ANCA-associated experimental autoimmune vasculitis (EAV), induced by immunization with human myeloperoxidase (MPO), was used to analyze directly the potential effect of ANCAs on leukocyte-venular wall interactions in vivo as observed by intravital microscopy. These rats developed anti-MPO antibodies directed against rat leukocytes, showed pathologic evidence of small vessel vasculitis, and had enhanced leukocyte adhesion and transmigration in response to the chemokine Gro $\alpha$ (CXCL1 [CXC ligand 1]). Passive transfer of immunoglobulin from rats with EAV to naive rats conferred enhanced adhesion and transmigration responses in the recipients. Furthermore, rats with EAV and recipients of ANCA-positive immunoglobu- lin developed extensive microvascular injury, as manifested by mesenteric hemorrhage, in response to CXCL1. This study provides the first direct in vivo evidence for the ability of ANCAs to enhance leukocyteendothelial interactions and cause microvascular hemorrhage, thereby providing a mechanism by which ANCAs could exert pathogenic effects in systemic vasculitis. (Blood. 2005;106:2050-2058)

๑ 2005 by The American Society of Hematology

\section{Introduction}

Systemic small vessel vasculitis (SVV) results in rapidly progressive glomerulonephritis and lung hemorrhage in humans and is usually fatal if untreated. ${ }^{1}$ It is characterized by microvascular inflammation and necrosis in a variety of organs. The 2 organs that are most extensively injured in this manner are the kidney, through the development of pauci-immune crescentic glomerulonephritis, and the lung, with consequent alveolar hemorrhage. The association between antineutrophil cytoplasm antibodies (ANCAs) and SVV, initially described in the $1980 \mathrm{~s},{ }^{2}$ has attracted considerable interest over the past decade. These autoantibodies are principally directed against myeloperoxidase (MPO) and proteinase-3. ${ }^{3,4}$ At first, ANCAs were regarded only as clinical markers of disease activity, but it is now apparent that they have direct biologic effects on neutrophils ${ }^{5,6}$ and monocytes. ${ }^{7}$ The binding of ANCAs to antigen expressed on the leukocyte cell surface following cytokine priming is followed by the activation of an array of intracellular signaling pathways, ${ }^{8}$ with resultant degranulation and dysregulated apoptosis. ${ }^{9}$

Granulocyte infiltration and fibrinoid necrosis of the vessel wall are the pathologic hallmarks of SVV. For this reason, investigative efforts have focused on the influence that ANCAs have on the interaction between leukocytes and the vascular endothelium, specifically with reference to the inflammatory cascade of leukocyte rolling, adhesion, and transmigration. In this context, in vitro studies using flow chamber models have shown that ANCAs can cause rolling neutrophils to arrest on platelet monolayers ${ }^{10}$ and to promote firm adhesion and migration of rolling neutrophils on endothelial cells. ${ }^{11,12}$ Despite growing in vitro evidence implicating ANCAs as an inducer of leukocyte-endothelial cell interactions, there has been no evidence to date demonstrating such an effect of these antibodies in vivo. The ability of ANCAs to induce systemic small vessel vasculitis and crescentic glomerulonephritis, however, has been demonstrated by transfer of anti-MPO antibodies in a mouse model of vasculitis. ${ }^{13}$ Crescent formation in these animals occurred secondary to fibrinoid necrosis of the glomerular tuft, which was induced by necrosis and occlusion of glomerular capillaries.

To investigate the mechanisms involved in the initiation of inflammatory microvascular injury, we have used a rat model of ANCA-associated SVV (AASV) that mimics the human condition, developed in our laboratory. ${ }^{14}$ In this model, experimental autoimmune vasculitis (EAV), Wistar-Kyoto (WKY) rats, immunized with purified human myeloperoxidase (hMPO) in complete Freund adjuvant (CFA), develop high titers of ANCAs, accompanied by pauci-immune crescentic glomerulonephritis, and lung hemorrhage. Because the aim of our study was to investigate the hypothesis that ANCAs are capable of promoting leukocyteendothelial interactions in vivo, leukocyte responses within the mesenteric vascular bed of immunized rats were directly investigated using intravital microscopy. The findings demonstrate
From The Eric Bywaters Centre for Vascular Inflammation, London, United Kingdom; the Renal Section, Division of Medicine, Imperial College London, Hammersmith Campus, London, United Kingdom; and the Department of Histopathology, Division of Medicine, Imperial College London, Hammersmith Campus, London, United Kingdom.

Submitted March 7, 2005; accepted May 18, 2005. Prepublished online as Blood First Edition Paper, June 2, 2005; DOI 10.1182/blood-2005-03-0921.

Supported by a Wellcome Trust Research Training Fellowship (M.A.L.), a National Kidney Research Fund Research Training Fellowship (C.L.S.), and by grants from the British Heart Foundation.

C.D.P. and S.N. are joint senior authors. M.A.L. and C.L.S. are joint first authors.
The online version of the article contains a data supplement.

An Inside Blood analysis of this article appears in the front of this issue.

Reprints: Charles Pusey, Renal Section, Division of Medicine, Imperial College London, Hammersmith Campus, London W12 0NN, United Kingdom; e-mail: c.pusey@imperial.ac.uk.

The publication costs of this article were defrayed in part by page charge payment. Therefore, and solely to indicate this fact, this article is hereby marked "advertisement" in accordance with 18 U.S.C. section 1734.

() 2005 by The American Society of Hematology 
increased leukocyte firm adhesion and transmigration, as induced by locally administered CXC ligand 1 (CXCL-1; a rat homologue of interleukin 8 [IL-8]), in rats immunized with hMPO, and in naive rats following passive transfer of ANCAs from rats with EAV. This was accompanied by microvascular injury, as shown by mesenteric hemorrhage in response to CXCL-1. Collectively, these results provide the first direct in vivo evidence that ANCAs can cause enhanced leukocyte-endothelial interactions and support the concept that ANCAs are pathogenic.

\section{Materials and methods}

\section{Animals}

WKY/NCrlBR rats were purchased from Charles River (Margate, United Kingdom). All animal studies were performed according to the directives of the United Kingdom Home Office Animals (Scientific Procedures) Act, United Kingdom (1986).

\section{Immunization protocol and characterization of EAV phenotype}

Rats (130-180 g) were immunized intramuscularly with hMPO (400, 800, and $1600 \mu \mathrm{g} / \mathrm{kg}$; Calbiochem, Merck Biosciences, Nottingham, United Kingdom; $n=13,11$, and 4, respectively) or human serum albumin (HSA; Sigma-Aldridge, Gillingham, United Kingdom; $\mathrm{n}=19$ ) in an equal volume of CFA. Hematuria was assessed with dipstick (Bayer Multistix, Berkshire, United Kingdom) and albuminuria with enzyme-linked immunosorbent assay (ELISA; Nephrat ELISA kit; Exocell, Philadelphia, PA). Anti-hMPO antibody levels in serum were measured by ELISA. Briefly, 96-well plates were coated overnight with hMPO $(2 \mu \mathrm{g} / \mathrm{mL})$ in carbonate buffer. Wells were then incubated with dilutions of serum samples in triplicate for 60 minutes at $37^{\circ} \mathrm{C}$, washed, and incubated with anti-rat immunoglobulin $\mathrm{G}$ (IgG) alkaline phosphatase conjugate (Sigma) for 45 minutes at $37^{\circ} \mathrm{C}$. Binding was detected with p-nitrophenylphosphate (p-NPP; Sigma) and read at $405 \mathrm{~nm}$.

Following intravital microscopy, rats were killed, and organs were fixed in formalin (for histologic analysis), snap-frozen in 22-oxacalcitriol (OCT; Miles, Elkhart, IN) (for immunofluorescence staining), and fixed in glutaraldehyde for analysis by electron microscopy. Glomerular crescent percentage was quantified blindly in 100 random glomeruli on each periodic acid Schiff-stained section. Images were captured with an Olympus BX40 microscope (Olympus Optical, London, United Kingdom) and a Photonic Science Color Coolview camera (Photonic Science, East Sussex, United Kingdom). Direct immunofluorescence staining for glomerular IgG deposition was performed on $3-\mu \mathrm{m}$ frozen sections after fixation in acetone for 10 minutes. Following washing in phosphate-buffered saline PBS, nonspecific binding was blocked by incubation with $20 \%$ normal rabbit serum, and sections were then incubated with fluorescein isothiocyanate (FITC)-conjugated anti-rat IgG (Sigma) for 60 minutes. To control for the thickness of the section, images were captured digitally using a laser scanning confocal microscope and Pascal LSM software (LSM5 Pascal; Zeiss, Jena, Germany).

\section{Ig preparation}

Ig from pooled rat sera of all actively immunized animals was prepared by $45 \%$ ammonium sulfate precipitation. Final Ig preparations had endotoxin levels of less than $5 \mathrm{EU} / \mathrm{mL}$, as measured by Limulus Amebocyte Lysate testing (Bio-Whittaker, Walkersville, MD). All Ig preparations were ultracentrifuged $\left(10^{5} \mathrm{~g}\right.$ for 30 minutes), and the presence of anti-hMPO antibodies in preparations from rats with EAV was confirmed using ELISA.

\section{Intravital microscopy}

Intravital microscopy on rat mesentery was performed as described previously. ${ }^{15}$ Briefly, control $(n=18)$ or EAV $(n=20)$ rats were anesthetized with intravenous sodium pentobarbitone, without the operator's knowledge of the immunization status of the rat, and maintained at $37^{\circ} \mathrm{C}$ on a heated microscope stage. Following midline abdominal incision, the mesentery adjoining the terminal ileum was carefully arranged over a glass window on the stage and superfused with Tyrode balanced salt solution. Baseline quantification of leukocyte rolling, firm adhesion, and transmigration was performed using a fixed stage upright microscope with water immersion objectives (Axioscope; Zeiss). To investigate the effect of recombinant rat CXCL1 (PeproTech, London, United Kingdom), the chemokine was continuously applied topically to the mesentery in the superfusion buffer (final concentration $3 \times 10^{-9} \mathrm{M}$ ), and leukocyte responses were quantified at regular intervals. In experiments investigating passive transfer of ANCAs, nonimmunized rats were injected in a blinded fashion with ANCA-containing $(\mathrm{n}=9)$ or control $(\mathrm{n}=8) \mathrm{Ig}(20 \mathrm{mg} / \mathrm{kg}$ intravenously).

In animals that developed extensive hemorrhage, each vessel segment (all of which had been chosen randomly before the addition of a stimulus) was assessed for the presence or absence of hemorrhage at each time point. The number of hemorrhagic venular segments was then expressed as a percentage of the total number of vessel segments observed. This quantification procedure was, however, insensitive for accurate recording of responses in rats with minor degrees of hemorrhage, as seen in the passive transfer experiments. In these, hemorrhage at the vessel segments chosen at the beginning of the experiment was infrequent. In these cases hemorrhage was quantified using a global visual-analog score, defined in dose-finding experiments as $0=$ no hemorrhage, $1=$ a single focus, $2=2$ to 5 foci, $3=6$ to 10 foci, $4=$ more than 10 foci with macroscopic petechiae visible, and $5=$ widespread hemorrhage with extensive microvascular occlusion. The experiments also typically involved measurement of peripheral differential leukocyte counts, blood pressure, and red blood cell (RBC) velocity, as previously detailed. ${ }^{16}$

\section{Assessment of anti-hMPO crossreactivity with neutrophils from WKY rats}

Binding of anti-hMPO antibodies to WKY rat neutrophils was demonstrated by indirect immunofluorescence staining, by flow cytometry, and by Western blotting of leukocyte lysates. With respect to fluorescence staining, neutrophil-rich leukocyte suspensions were prepared from healthy human volunteers and nonimmunized WKY rats by discontinuous density centrifugation. Smears were air-dried and fixed in $95 \%$ ethanol before incubation with serum (1:20 in PBS) from EAV $(n=13)$ or control $(n=10)$ rats, or plasma (1:20 in PBS) from anti-MPO antibody-positive patients (microscopic polyangiitis, $n=5$ ) or anti-MPO-negative controls (IgA nephropathy, $\mathrm{n}=2$; antibody-mediated transplant rejection, $\mathrm{n}=1$ ). In addition, staining following serial dilution of selected sera to 1:320 was performed. Binding of antibodies was detected using appropriate Alexa Fluor 568conjugated anti-rat/human IgG antibodies (Molecular Probes, Eugene, OR). Samples were observed using a laser scanning confocal microscope (LSM5 Pascal; Zeiss) with the aid of Pascal LSM software. Fluorescence intensity was quantified using Image-Pro plus software (v3.0; Media Cybernetics, Silver Spring, MD).

For flow cytometry, mixed leukocyte preparations from WKY rats and healthy human volunteers were prepared by sedimentation of red cells using 2.5\% Dextran T-500 (Amersham Biosciences, Chalfont, United Kingdom) with permeabilization of cell samples with Saponin ( $0.1 \%$ in PBS). Surface and intracellular antigen expression was detected with Ig preparations from EAV or control rats $(100 \mu \mathrm{g} / \mathrm{mL}$ in $\mathrm{PBS} / 20 \%$ rabbit serum) and a FITC-conjugated rabbit anti-rat IgG (Sigma). In selected experiments granulocytes were distinguished by double staining with phycoerythrin (PE)-conjugated HIS48 IgM (Santa Cruz Biotechnology, Santa Cruz, CA). The samples were acquired on a FACSCalibur flow cytometer (Becton Dickinson, Franklin Lakes, NJ). Binding of EAV Ig relative to control Ig was expressed as mean ratio of mean fluorescence intensity (RFI).

For Western blot analysis, mixed leukocytes were prepared as for flow cytometry, lysed, and run on sodium dodecyl sulfate-polyacrylamide gel electrophoresis (SDS-PAGE). Briefly, after lysing residual RBCs with ice-cold $\mathrm{NH}_{4} \mathrm{Cl}$ lysis solution, the leukocyte pellet was suspended in lysis buffer (1\% Triton X-100, $10 \mathrm{mM}$ Tris [tris(hydroxymethyl)aminomethane], $5 \mathrm{mM}$ EDTA [ethylenediaminetetraacetic acid], $50 \mathrm{mM} \mathrm{NaCl}, 30 \mathrm{mM}$ $\mathrm{Na}_{4} \mathrm{O}_{7} \mathrm{P}_{2}, 50 \mathrm{mM} \mathrm{NaF}, 100 \mathrm{mM} \mathrm{Na} \mathrm{VO}_{4}, \mathrm{pH}$ 7.6) containing protease 
inhibitors (Sigma) and incubated on ice for 20 minutes. The samples were centrifuged at $14000 \mathrm{~g}$ for 20 minutes at $4^{\circ} \mathrm{C}$, and nondenaturing SDSPAGE was performed on the lysate supernatant with a Phast-gel system using $7.5 \%$ agarose gels (Amersham Pharmacia Biotech, Uppsala, Sweden). The purified hMPO $(50 \mu \mathrm{g} / \mathrm{mL})$ used to induce EAV and lysates of human polymorphonuclear leukocytes (PMNs) purified over Percoll were used as positive controls. Samples were transferred to nitrocellulose membranes and incubated with primary antibodies (Ig preparations from rats with EAV and control rats), followed by incubation with appropriate anti-rat IgG alkaline phosphatase conjugates, with all incubations being for 60 minutes. Binding was detected using BCIP/NBT (5-bromo-4-chloro-3indolyl-phosphate, 4-nitroblue tetrazolium chloride) alkaline phosphatase substrate (Sigma).

\section{Statistical analysis}

Normally distributed data are expressed as the mean plus or minus SEM, and non-normal data are expressed as median and interquartile range (IQR). Baseline variables were compared using Student $t$ test, and urinary albumin excretion rate and hemorrhage at 90 minutes was analyzed with the Mann-Whitney test. Intravital microscopy adhesion and transmigration data were analyzed using 2-way analysis of variance (ANOVA), considering time and treatment group as independent variables. Values at individual time points were compared using Bonferroni post hoc tests.

\section{Results}

\section{Immunization of WKY rats with hMPO induces anti-hMPO} antibodies that bind to WKY rat neutrophils

To assess the effect of circulating ANCAs on microvascular leukocyte-endothelial responses, WKY rats were immunized with hMPO (EAV group) or HSA (control group) in CFA. As there were no differences in any of the outcome variables studied between the $3 \mathrm{hMPO}$ dosing groups used $(400,800$, and $1600 \mu \mathrm{g} / \mathrm{kg})$, for all analyses the groups receiving different doses of hMPO were considered together. Because binding of anti-hMPO antibodies to rat neutrophils was critical to our in vivo investigations, initial experiments evaluated this using a number of techniques.

Using ELISA, all rats immunized with hMPO had titers of anti-hMPO antibodies in excess of 1:1000, and Ig preparations from rats with EAV had detectable anti-hMPO activity at concentrations above $0.5 \mu \mathrm{g} / \mathrm{mL}$. Rats immunized with HSA, or Ig prepared from these rats, had no detectable anti-hMPO activity. As EAV was induced with human antigen, it was essential to assess the ability of ANCAs from rats with EAV to bind to rat MPO (and rat neutrophils) as a prerequisite for demonstrating any potential biologic effect. This was established by immunofluorescence staining, by flow cytometry, and by Western blot analysis of neutrophil lysates.

Observation of ethanol-fixed rat and human neutrophils by confocal microscopy (Figure 1A), after indirect staining with various sera, indicated that anti-hMPO antibody-positive sera from EAV rats bound to WKY neutrophils in a cytoplasmic pattern, whereas they bound to human neutrophils in a typical perinuclear pattern. ${ }^{17}$ The pattern of binding of EAV rat sera to human or rat neutrophils was similar to the pattern produced by human MPOANCA-positive sera from patients with vasculitis on the same substrates. Fluorescence intensity on WKY cells remained above that obtained with anti-hMPO-negative serum up to a dilution of 1:320. The intensity of binding of rat anti-hMPO-positive serum to rat neutrophils was less than that observed with human neutrophils but consistently greater than that produced by anti-hMPO antibodynegative sera. These observations are in agreement with Image-Pro quantification of fluorescence intensity (Figure 1B) and with flow cytometry results confirming the binding of Ig from EAV rats (but not control Ig) to rat and human granulocytes (Figure 1C) with RFI values of 2.0 and 6.9 , respectively ( $\mathrm{n}=4$ separate experiments). Considering the cell population staining positive for the rat granulocyte marker, HIS48, the percentage of cells staining positive for FITC increased from 14\%, when incubated with control Ig, to $72 \%$ when incubated with EAV Ig.

The binding of Ig preparations from hMPO immunized and control rats to WKY and human neutrophils was also investigated by Western blotting (Figure 1D). Immunoglobulin preparations from rats with EAV bound to several bands in the purified hMPO and human leukocyte lanes, the strongest being at $126 \mathrm{kDa}$ and 74 $\mathrm{kDa}$, and to a single $120-\mathrm{kDa}$ band in the WKY rat leukocyte lane. Under denaturing conditions, this band was at $59 \mathrm{kDa}$, suggesting that it is the heavy chain of MPO. No binding was observed to either human or WKY lysates using Ig from control rats. Collectively, these findings confirm that the polyclonal antibodies that develop in hMPO immunized EAV rats bind to antigen in WKY rat neutrophils.

\section{Immunization of WKY rats with hMPO induces pauci-immune crescentic glomerulonephritis and pulmonary hemorrhage}

Gross examination of lungs and kidney revealed petechiae over the lung surface, and occasional petechiae on the renal surface in rats with EAV, but not in control rats. Prominent macroscopic lung hemorrhage was evident in $41 \%$ of rats immunized with hMPO. In all of these, there was histologic evidence of lung vasculitis, with perivascular leukocyte cuffing, occasional vascular occlusion, and alveolar hemorrhage (Figure 2). In $79 \%$ of hMPO-immunized animals, but none of the control animals, focal proliferative glomerulonephritis with fibrinoid necrosis was evident (Figure 2). In $78 \%$ of these ( $61 \%$ of all hMPO-immunized rats), crescent formation was present (Figure 2). This was focal, with adjacent areas of normal renal tissue, and often associated with foci of tubulo-interstitial nephritis. Immunofluorescence examination of renal sections revealed occasional scanty deposits of $\mathrm{IgG}$. The amount of deposits was above the background level seen with sections from animals immunized with CFA alone, but much less than that seen with rats with experimental autoimmune glomerulonephritis, a model of antiglomerular basement membrane disease (Figure 3). Overall mean percentage of glomeruli with crescents in rats with EAV was $5.2 \% \pm 1.6 \%$ (Figure 4). Consistent with the histologic findings, no immune deposits were evident on samples analyzed by electron microscopy (data not shown). All EAV rats, but no control rats, developed hematuria and $72 \%$ had albuminuria 6 weeks after immunization $(>1 \mathrm{mg} / 24$ hours, mean $11.9 \pm 4.4$ $\mathrm{mg} / 24$ hour; Figure 4).

\section{Induction of ANCAs in WKY rats augments leukocyte adhesion and transmigration induced by CXCL1}

Having demonstrated the presence of ANCAs in our rat model of $\mathrm{EAV}$, their effects on leukocyte-endothelial interaction in vivo were investigated by intravital microscopy using the mesenteric preparation. For this purpose, leukocyte responses within rat mesenteric venules were observed and quantified in EAV rats in the absence of an inflammatory stimulus and after topical application of the 
Figure 1. Sera from hMPO and HSA-immunized WKY rats bind to WKY and human neutrophils. (A) Human and WKY rat PMNs were indirectly stained with sera $(1: 20)$ from hMPO (EAV) or HSA-immunized (control) rats, and sera from patients with AASV and observed by confocal microscopy $(\times 63$; UplanApo $63 \times / 1.20$ water dipping). Representative of 5 experiments involving 5 to 13 separate sera. (B) Cells were stained as in panel $A$, and fluorescence intensity was quantified using Image-Pro plus software. Circles indicate anti-hMPO-positive sera; triangles, anti-hMPOnegative sera. Statistically significant differences are shown by asterisks, ${ }^{* *} P<.01$ and ${ }^{* * *} P<.001$. AFU indicates arbitrary fluorescence unit. (C) Rat leukocytes were incubated with Ig prepared from EAV rats (open curves) or control rats (filled curves). Both surface and intracellular antigens were stained sequentially, and rat PMNs were identified based on side scatter profile and binding of anti-HIS48 monoclonal antibody (mAb). Panels $\mathrm{Ci}$ and $\mathrm{Cii}$ represent cells stained with control Ig (closed curves) or EAV Ig (open curves) on rat and human PMNs, respectively. Results are representative of 4 separate experiments. (D) Lysates of WKY rat and human leukocytes were prepared and analyzed by Western blotting. (Lane 1) Purified hMPO incubated with pooled Ig from rats with EAV. (Lane 2) WKY rat leukocyte lysate incubated with pooled Ig from rats with EAV. (Lane 3) WKY rat leukocyte lysate incubated with pooled Ig from control rats. (Lane 4) Human PMN lysate incubated with pooled Ig from rats with EAV. Separate blots are indicated by vertical lines.
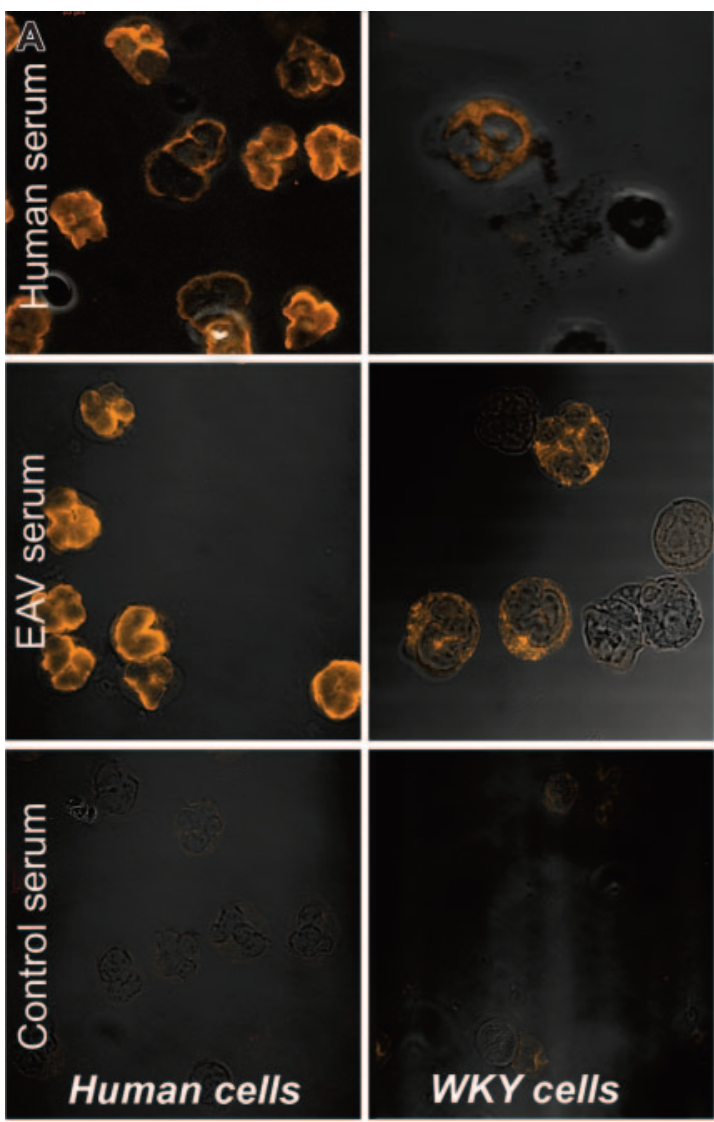

B
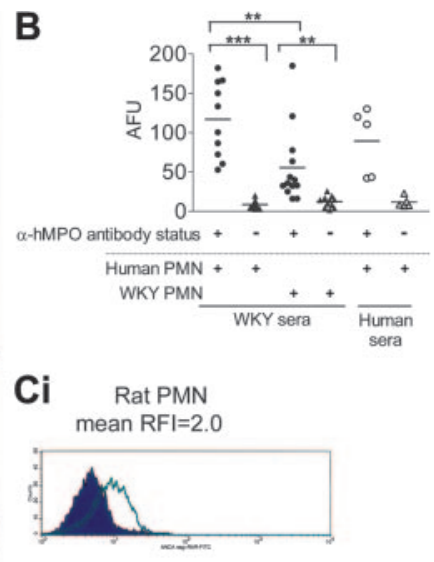

Cii

mean $\mathrm{RFI}=6.9$

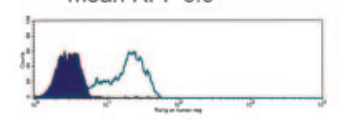

D

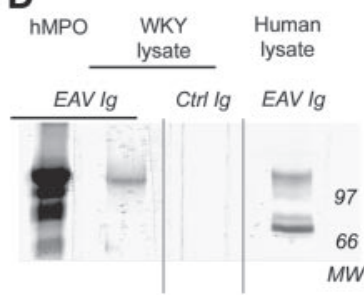

chemokine CXCL1. CXCL1 is a rat homologue of IL-8, a chemokine which is likely to be of pathophysiologic relevance in ANCA-associated vasculitis. ${ }^{18}$ Intravital microscopy was performed on rats 6 to 7 weeks after immunization with hMPO or HSA. Of note, there was no significant difference between the 2 groups of rats with respect to multiple systemic or microhemodynamic parameters (Table 1). Furthermore, no significant difference in basal leukocyte rolling flux or leukocyte transmigration was noted between the 2 groups of rats. Baseline firm adhesion was variable and slightly higher in the HSA-immunized group as compared with the hMPO-immunized group. Although the reason for this small difference is unclear, overall the findings do not indicate the occurrence of increased leukocyte-vessel wall interactions in the EAV rats under basal conditions.

In contrast, after topical CXCL1 application, a significant increase in both leukocyte firm adhesion and transmigration was observed in hMPO-immunized compared with HSA-immunized rats (Figure 5). Sixty minutes after application of the chemokine, adhesion was increased by $32 \%$ in the hMPO-immunized group $(P<.01)$, a response that was accompanied by a $65 \%$ increase in transmigration at 90 minutes $(P<.001)$. There was no correlation between the severity of glomerular injury and the intravital microscopy findings. Thus, in an acute inflammatory milieu, as induced in the present study by local application of the chemokine CXCL1, hMPO-immunized rats, with high titers of circulating ANCAs, exhibited enhanced microvascular inflammatory responses of leukocyte adhesion and transmigration. These findings provide in vivo evidence of a potential pathophysiologic effect of ANCAs in EAV, a model with pathology that very closely mimics human ANCA-associated SVV.

\section{Passive transfer of ANCA-rich Ig confers enhanced microvascular responses to $\mathrm{CXCL1}$ in naive recipient rats}

To confirm that the enhanced microvascular responses observed in rats with EAV were due to circulating ANCAs, Ig prepared from sera from hMPO- or HSA-immunized rats was transferred to naive WKY rats prior to quantification of leukocyte responses by intravital microscopy. The protocol used was similar to that described in the previous section, with the addition of a 30-minute quantification period following Ig injection prior to mesenteric superfusion with CXCL1 or Tyrode solution.

Injection of either EAV or control $\mathrm{Ig}(20 \mathrm{mg} / \mathrm{kg})$ had no effect on baseline systemic or microhemodynamic variables (Table 1). Before Ig infusion, adhesion and transmigration levels were not significantly different between the 2 groups. However, following intravenous injection of EAV Ig, but not control Ig, leukocyte firm adhesion was significantly enhanced in rats treated with topical Tyrode solution (mean adhesion at 120 minutes after EAV and control Ig were $6.6 \pm 2.1$ and $0.6 \pm 0.2$ leukocytes/100 $\mu \mathrm{m}$ segment, respectively; $\mathrm{n}=3-4 ; P<.001$; Figure 6A). Interestingly, this increased adhesion response was not accompanied by an increase in leukocyte transmigration (Figure 6B). These results demonstrate that, using the present experimental protocol, ANCArich Ig on its own can induce leukocyte firm adhesion to venular walls, although this enhanced leukocyte-endothelial cell interaction does not lead to leukocyte transmigration. Hence, in light of our findings in the active immunization model, we next investigated the effect of intravenous ANCA-rich Ig on leukocyte responses induced by topical CXCL1. In agreement with our previous results, topical administration of this chemokine elicited time-dependent increases in both leukocyte firm adhesion and transmigration in rats 


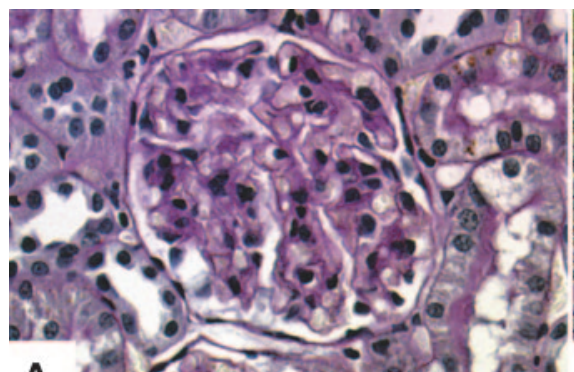

A

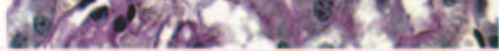
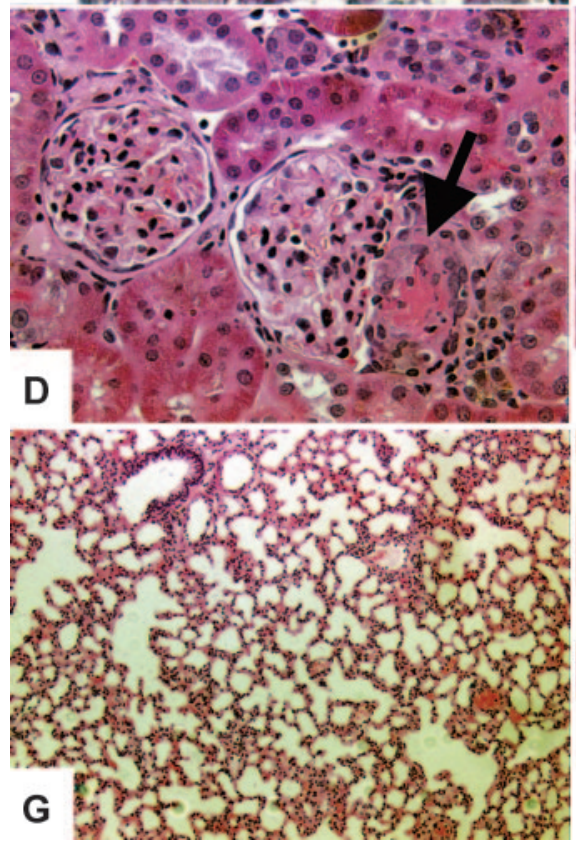

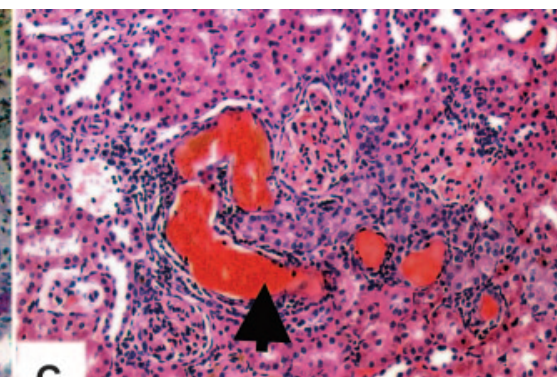

C
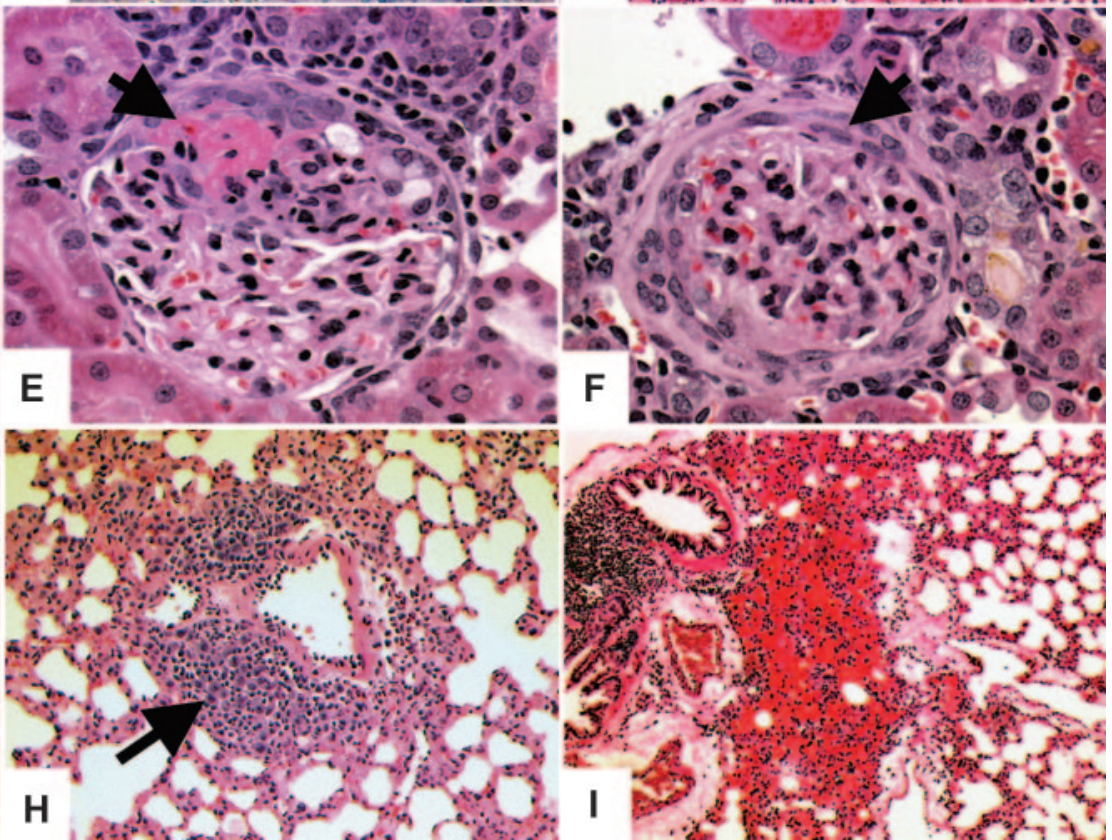

Figure 2. WKY rats immunized with hMPO develop focal necrotizing crescentic glomerulonephritis and pulmonary hemorrhage. (A) No histologic abnormalities in a rat immunized with HSA (Periodic acid Schiff; UplanApo 40×/0.85 NA); (B) severe crescentic glomerulonephritis (arrows) in a rat immunized with hMPO (Periodic acid Schiff; UplanApo $10 \times / 0.40 \mathrm{NA}$ ); (C) tubulo-interstitial nephritis with red cell cast (arrow) in a rat immunized with hMPO (hematoxylin and eosin [H\&E]; UplanApo 10×/0.40 NA); (D) focal glomerular necrosis, with an adjacent glomerulus showing normal architecture in a rat immunized with hMPO (H\&E; UplanApo $20 \times / 0.70 \mathrm{NA}$ ); (E) focal necrotizing lesion with early crescent formation (arrow) in a rat immunized with hMPO (H\&E; UplanApo 40×/0.85 NA); (F) circumferential glomerular crescent (arrow) in a rat immunized with hMPO (H\&E; UplanApo 10×/0.40 NA); (G) normal lung architecture in a rat immunized with HSA (H\&E; UplanApo 10×/0.40 NA). (H) Pulmonary vasculitis (arrow) in a rat immunized with hMPO (H\&E; UplanApo 20×/0.70 NA); (I) pulmonary hemorrhage in a rat immunized with $\mathrm{hMPO}$, with extensive blood in the alveolar spaces (H\&E; UplanApo $10 \times / 0.40 \mathrm{NA}$ ).

injected with control Ig, as compared with baseline values (Figure $6 \mathrm{C}-\mathrm{D})$. Both of these responses were enhanced in rats receiving ANCA-rich Ig, with the transmigration response at 90 minutes after application of CXCL1 being $79 \%$ greater in rats injected with EAV $\mathrm{Ig}$, as compared with rats injected with control Ig.

Taken together, these findings demonstrate that the MPOANCAs induced in the EAV rat model of SVV have a biologic effect on leukocyte adhesion and transmigration in vivo. To address the association of ANCAs with vascular injury, the experimental models detailed in the previous two sections were extended to enable direct investigation of microvascular injury resulting in hemorrhage.

\section{Transfer of ANCA can induce microvascular hemorrhage}

Patients with SVV develop microvascular occlusion and destruction with consequent hemorrhage into surrounding tissue. This is manifested by purpura, lung hemorrhage, and bleeding into other organs. Hence, the effect of ANCAs on the hemorrhagic response to an inflammatory stimulus was of particular interest. To quantify
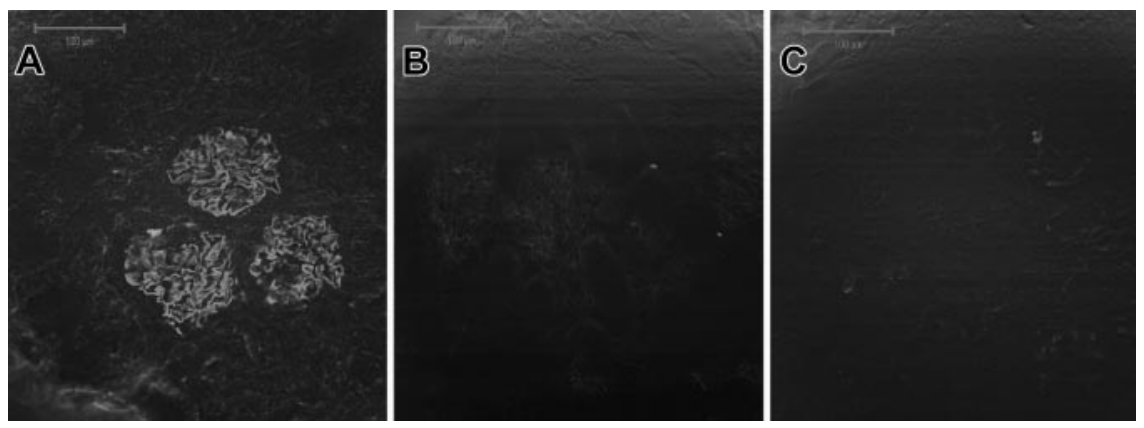

Figure 3. Glomerulonephritis in EAV is pauciimmune. Glomerular Ig deposition was assessed using direct immunofluorescence on 3- $\mu \mathrm{m}$ frozen sections. (A) Linear staining for $\operatorname{lgG}$ in a rat with experimental autoimmune glomerulonephritis, a model of antiglomerular basement membrane disease. This serves as a positive control; (B) scanty Ig deposits in a rat with EAV; (C) negative staining in a rat immunized with CFA alone. Images were captured using a confocal microscope, and bars represent $100 \mu \mathrm{m}(\times 20)$. 
Figure 4. EAV is characterized by hematuria, lowgrade albuminuria, and focal crescent formation. Six weeks after immunization, hematuria $(A)$ was assessed by dipstick and albuminuria (B) by ELISA. (C) The percentage of glomeruli with crescents was quantified on Periodic acid Schiff sections. ${ }^{* \star *} P<.001$ comparing proteinuria between EAV and control rats. No statistical comparison is possible in panels $A$ and $C$ because all values in the control group are zero.
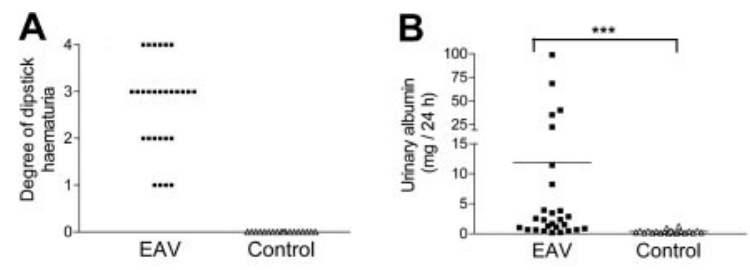

this variable by intravital microscopy, 2 methods were used, governed by the extent of the response. In actively immunized rats, where the hemorrhagic response was relatively extensive and focal sites of hemorrhage could be seen by naked eye (Figure 7A), or by histologic analysis (Figure 7B), the response was quantified as a fraction of responding venular segments. Using this approach, a trend toward an increase in hemorrhagic response following superfusion with CXCL1 was observed in hMPO-immunized rats (17.1\%; interquartile range of $0-37$ in EAV versus $0 \%$, interquartile range of $0-15$ in control rats; $P=.09$; Figure $7 \mathrm{C}$ ). For quantification of minor degrees of hemorrhage, as observed in the passive transfer experiments, a global visual-analog score (0-5) was used to assess the severity of hemorrhage in the mesentery as a whole. Using this method, recipients of ANCA-rich Ig showed a significant increase in microvascular hemorrhagic response following topical application of CXCL1 (Figure 7D), with a trend toward spontaneous hemorrhage following superfusion with Tyrode solution alone. Real-time development of ANCA-induced microvascular hemorrhage is depicted in Video S1 (available at the Blood website; see the Supplemental Video link at the top of the online article).

\section{Discussion}

The presence of ANCAs is implicated in the pathogenesis of SVV and has been shown in murine models to be sufficient to cause pauci-immune glomerulonephritis. ${ }^{13}$ However, to date, there has been no in vivo evidence directly associating these antibodies with leukocyte-mediated vascular damage. With the use of a novel rat model of ANCA-driven EAV, which has many similarities to human ANCA-associated vasculitis, our findings provide the first direct evidence for the ability of ANCAs to enhance leukocytevessel wall interactions in vivo. Specifically, rats with EAV exhibited an exaggerated adhesion and transmigration response to a pathophysiologically relevant inflammatory stimulus, CXCL1, as observed by intravital microscopy. This effect was transferable to naive rats by transfer of $\mathrm{Ig}$, proving that the effect was due to circulating ANCAs. Indeed, ANCAs on their own, without any additional topical stimulus, was capable of inducing firm adhesion of leukocytes to the vascular endothelium. Furthermore, our study provides evidence to support the concept that ANCA-induced exaggerated leukocyte responses can lead to vascular injury, as manifested by the development of microvascular hemorrhage. It is possible that these are early events in the cascade leading to necrotizing vasculitis.

WKY rats immunized with hMPO develop a polyclonal antibody response to this antigen that is accompanied by the development of pauci-immune focal necrotizing crescentic glomerulonephritis and pulmonary hemorrhage. This animal model of autoimmune SVV is similar in many respects to the analogous human disease and provides a unique opportunity to study the effects of ANCAs in vivo. Specifically, the glomerular lesion seen, although relatively mild, is pauci-immune in nature, as demonstrated by immunofluorescence and electron microscopy; that is there are only scant immune deposits present. The EAV model, like human AASV, is primarily a hematuric condition. Although albuminuria is present in the majority of rats with EAV, it is mild (around 3-10 mg/24 hour) compared with other rat models of glomerulonephritis. ${ }^{19}$ This model has some similarities with previous work performed in the early 1990s. Brouwer et $\mathrm{al}^{20}$ used heterologous MPO (at a low dose, $10 \mu \mathrm{g} / \mathrm{rat}$ ) to induce anti-MPO antibodies in Brown Norway rats. These animals developed granulomatous crescentic nephritis in kidneys perfused with lysosomal extract and hydrogen peroxide. Although immune deposits were initially felt to be transient in this model, Yang et $\mathrm{al}^{21}$ found deposits up to day 10. Although many researchers now feel that this is a model of immune-complex deposition disease, rather than pauci-immune vasculitis, it provided an important foundation for future work.

Although the protein sequences of rat (accession gi27674721) and human (accession gi4557759) MPO share 85.7\% homology, ${ }^{22}$ previous studies using an ELISA-based approach have found that monoclonal anti-hMPO antibodies raised in mice, and sera from patients with AASV, have limited crossreactivity with rat MPO. ${ }^{23}$ Because of these reports, and because the binding of rat anti-hMPO antibodies to rat leukocytes was critical to our in vivo functional studies, initial experiments investigated this crossreactivity. Evidence was provided for the ability of anti-hMPO antibodies raised in rats to bind to rat leukocytes. We believe that the difference

Table 1. Mean arterial pressure, leukocyte count, blood flow, and vessel diameter in test rats

\begin{tabular}{|c|c|c|c|c|c|c|c|}
\hline & \multicolumn{2}{|c|}{ Mean arterial pressure, $\mathrm{mm} \mathrm{Hg}$} & \multicolumn{2}{|c|}{ Leukocyte count, $\times 10^{9} / \mathrm{L}$} & \multicolumn{2}{|c|}{ Mean blood flow, $\mathrm{mm} / \mathrm{sec}$} & \multirow{2}{*}{$\begin{array}{c}\text { Vessel } \\
\text { diameter, } \mu \mathrm{m}\end{array}$} \\
\hline & Base & $90 \mathrm{~min}$ & PMN & PBMC & Base & $60 \mathrm{~min}$ & \\
\hline \multicolumn{8}{|c|}{ Active immunization } \\
\hline EAV & $105.6 \pm 3.9$ & $109.8 \pm 5.8$ & $1.5 \pm 0.1$ & $5.7 \pm 0.5$ & $1.1 \pm 0.1$ & $1.8 \pm 0.2$ & $28.7 \pm 0.4$ \\
\hline Control & $103.4 \pm 2.6$ & $106.9 \pm 3.0$ & $1.2 \pm 0.2$ & $5.8 \pm 0.7$ & $1.5 \pm 0.3$ & $2.1 \pm 0.4$ & $28.0 \pm 0.4$ \\
\hline \multicolumn{8}{|c|}{ Passive transfer } \\
\hline $\mathrm{ANCA}^{+}$ & $105.4 \pm 3.0$ & $121.4 \pm 3.7$ & $1.0 \pm 0.1$ & $4.1 \pm 0.4$ & ND & $3.4 \pm 0.6$ & $30.3 \pm 0.7$ \\
\hline $\mathrm{ANCA}^{-}$ & $106.0 \pm 3.8$ & $118.3 \pm 2.7$ & $1.3 \pm 0.3$ & $4.0 \pm 0.4$ & ND & $3.1 \pm 0.4$ & $30.0 \pm 0.6$ \\
\hline
\end{tabular}

WKY rats were immunized ("Active immunization") with hMPO (EAV, $n=7-20$ per group) or HSA (Control, $n=8-18$ per group) in CFA, or received Ig (“Passive transfer"; $\mathrm{ANCA}^{+}, \mathrm{n}=9, \mathrm{ANCA}^{-}, \mathrm{n}=9$ ). No significant differences between the groups within each experiment were observed. Values are mean $\pm \mathrm{SEM}$.

ND indicates not done. 

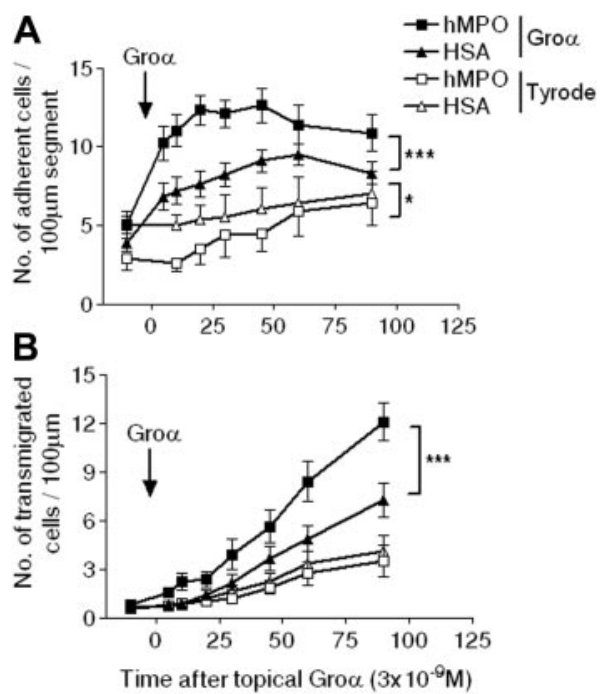

Figure 5. Adhesion and transmigration profiles in WKY rats actively immunized with hMPO or HSA in response to topical CXCL1. Intravital microscopy was performed on rats 6 to 7 weeks after immunization with hMPO (EAV rats) or HSA (control rats). After recording baseline responses of adhesion (A) and transmigration (B), topical CXCL1 $\left(3 \times 10^{-9} \mathrm{M}\right)$ or Tyrode balanced salt solution was superfused over the mesentery, and responses were quantified for a further 90 minutes, during which time superfusion of the topical agent was maintained. The data represent mean \pm SEM, $n=11$ to 13 separate rats per group for $C X C L 1$ and $n=6$ to 7 per group for Tyrode solution. Statistically significant differences between different groups of rats are shown by asterisks, ${ }^{*} P<.05$ and ${ }^{* \star *} P<.001$.

between our findings and those in the study by Patry et $\mathrm{al}^{23}$ relate to 2 factors. First, in the majority of their experiments, monoclonal antibodies were used, which presumably have activity against a restricted number of epitopes on the MPO molecule. We have induced high titers of polyclonal antibodies, among which there are apparently antibodies that crossreact with epitopes on the rat MPO molecule. Second, in the previous study, rat MPO was immobilized on plastic, which may alter the conformational epitopes compared with those found in the cellular systems that we used.

Leukocyte-vessel wall interactions in EAV rats, as compared with HSA-immunized control rats, were investigated in mesenteric venules as observed by intravital microscopy. Using this model, topical application of the chemokine CXCL1 induced a significant increase in leukocyte firm adhesion and transmigration in control rats, compared with rats treated with topical Tyrode solution. These responses were significantly enhanced in rats immunized with hMPO, resulting in a $65 \%$ increase in leukocyte transmigration in EAV rats 90 minutes after superfusion of the mesenteric tissue with CXCL1. Of note, CXCL1 is a rat homologue of the human chemokine IL-8 (CXCL8), an inflammatory mediator implicated in the pathogenesis of ANCA-associated vasculitis. ${ }^{18}$ The need for a priming factor to observe potentially pathogenic effects of ANCA on neutrophils is consistent with numerous previous in vitro studies. Priming with cytokines such as tumor necrosis factor $\alpha$ $(\mathrm{TNF} \alpha)$ has been shown to be necessary to facilitate the stimulation by ANCAs of enhanced neutrophil degranulation and neutrophildependent endothelial cytotoxicity. ${ }^{5,24}$ In addition, although the microvessels of the mesentery are not identical to those of the glomerular tuft, there is evidence to suggest that endothelial dysfunction seen in AASV is global. ${ }^{25}$ Thus, our findings demonstrate that the presence of ANCAs have the capacity to enhance leukocyte-vessel wall interactions as induced by a pathologically relevant inflammatory mediator.

To assess directly the role of circulating ANCAs in these observations, ANCA-rich Ig were prepared from the serum of EAV rats and administered to naive animals. Of note, it is not possible to compare directly the responses observed in these passive transfer experiments to those in the actively immunized animals, because the use of Freund adjuvant in the latter was associated with enhancement of all leukocyte-endothelial responses. Naive rats injected intravenously with ANCA-rich Ig exhibited a rapid and significantly enhanced leukocyte firm adhesion response that was not observed in rats injected with control Ig. Interestingly, this increase in leukocyte firm adhesion was not associated with enhanced transmigration, suggesting that perhaps a second inflammatory stimulus was required to induce the migration of adherent leukocytes through venular walls. Indeed, topical application of CXCL1 to mesenteric tissue of rats previously injected with intravenous ANCA-rich Ig led to a significantly enhanced transmigration response, as compared with responses detected in naive rats injected with Ig from HSA-immunized rats. Taken together, these findings provide the first direct evidence for the ability of ANCAs
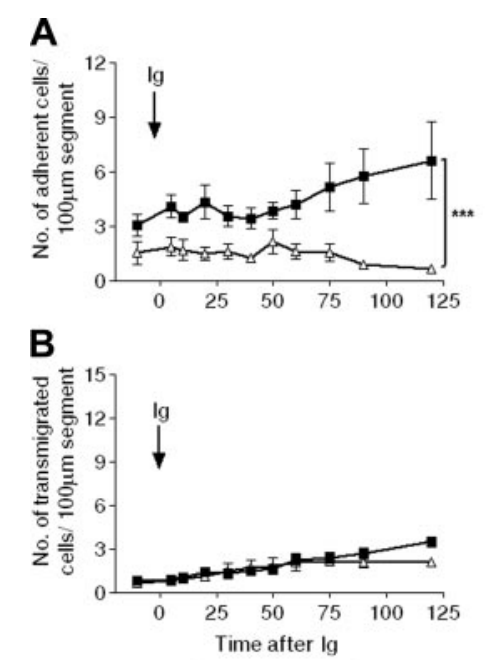

Tyrode alone

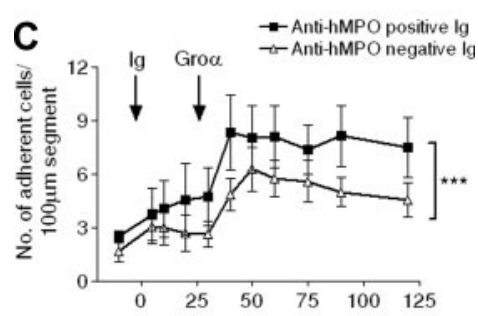

D

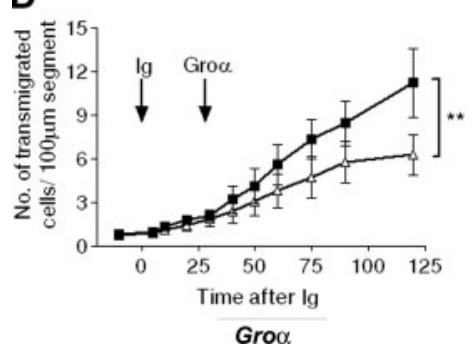

Figure 6. Passive transfer of ANCA-rich immunoglobulin enhances microvascular responses in naive WKY rats. Intravital microscopy was performed on naive WKY rats following intravenous administration of $20 \mathrm{mg} / \mathrm{kg}$ pooled anti-hMPOpositive $\lg (\bullet)$ or control $\lg (\triangle)$, and leukocyte responses of adhesion $(A, C)$ and transmigration (B,D) were measured for up to 2 hours. In some rats, after 30 minutes, the Tyrode mesenteric superfusion was changed to superfusion with $3 \times 10^{-9} \mathrm{M}$ CXCL1 (C,D), which was maintained for a further 90 minutes. The data represent the mean \pm SEM, $n=5$ separate rats in CXCL1 groups and $n=3$ to 4 in Tyrode groups. Statistically significant differences between groups of rats are shown by asterisks, ${ }^{\star \star} P<.01$, and ${ }^{\star \star *} P<.001$. 
Figure 7. ANCAs induce postcapillary venular hemorrhage. Intravital microscopy was performed on WKY rats 6 to 7 weeks after immunization with hMPO (EAV rats) or HSA (control rats) (C) or on naive WKY rats with infusion of ANCA-rich or ANCA-negative Ig (D). A representative macroscopic image of petechiae around a mesenteric arcade is shown in panel $A$ ( $\times 4$; image captured with a Canon IXUS 400; Canon, Tokyo, Japan). The microscopic appearance of this is shown in panel B (H\&E stain, UplanApo $20 \times 10.70 \mathrm{NA}$ ). In the active immunization model (C), superfusion with $3 \times 10^{-9} \mathrm{M} \mathrm{CXCL} 1$ was maintained for 90 minutes, and hemorrhage was quantified by expressing the number of hemorrhagic venular segments as the percentage of total segments studied at each time point. The data represent the median, interquartile range (box), and range (error bars, $\mathrm{n}=11$ in control group and 13 in EAV group). Of note, although minor degrees of hemorrhage were seen in the control group, the median remained at zero throughout the experiment. In the passive transfer model (D) superfusion fluid was changed in some experiments from Tyrode solution to CXCL1 30 minutes after lg infusion, and hemorrhage was quantified after a further 90 minutes using a global mesenteric visual/ analog score ( $n=4-10$ separate rats in each group). Statistically significant differences between groups of rats are shown by asterisks, ${ }^{\star} P<.05$. Data in panels $C$ and $D$ are mean \pm SEM.

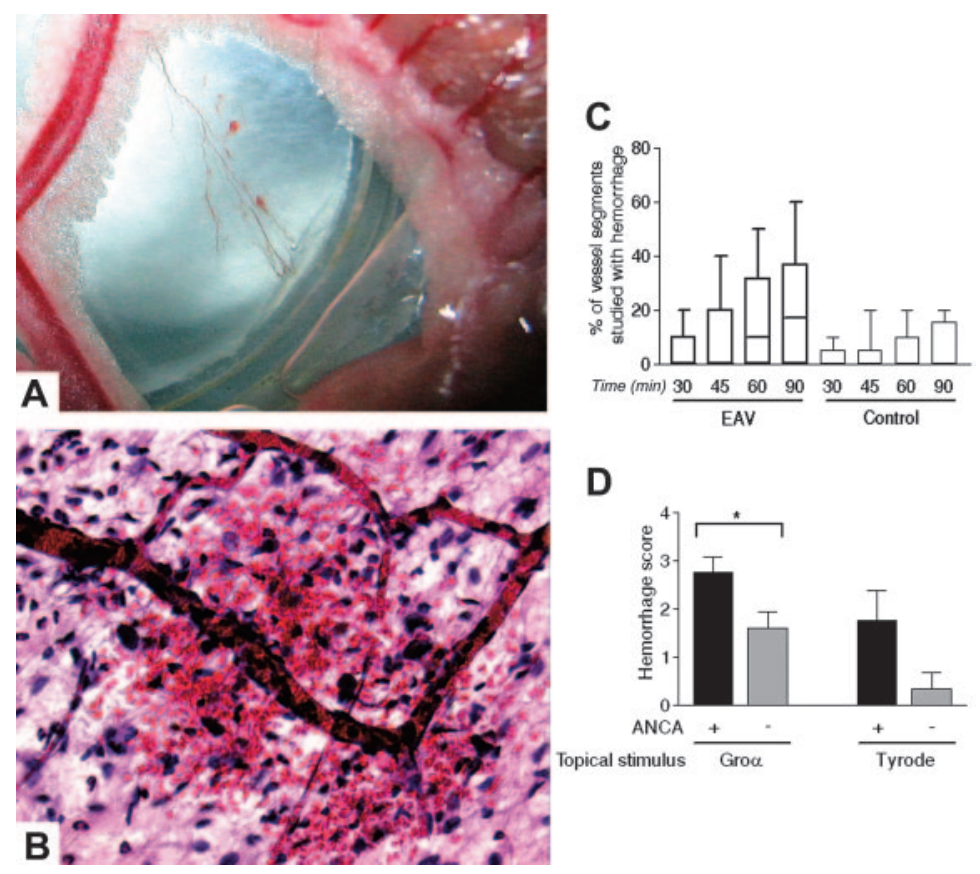

to enhance leukocyte firm adhesion, a response that under conditions of a local inflammatory milieu (as induced in our model by topical application of CXCL1) can be translated into leukocyte transmigration.

While the precise mechanism by which this occurs in vivo is at present unclear, in vitro studies have provided a number of potential mechanistic indications. For example, the binding of ANCAs to leukocytes has been shown to stimulate activation and/or up-regulation of $\beta_{2}$ integrins. ${ }^{26,27}$ Indeed, as illustrated by Radford et al, ${ }^{11}$ under both static and flow conditions, ANCAs can markedly increase neutrophil adhesion to untreated and stimulated endothelium ${ }^{28,29}$ in a $\beta_{2}$ integrin-dependent manner. Hence, we can speculate that, within the in vivo scenario of our studies, binding of ANCAs to neutrophils renders them primed for an enhanced adhesion response. In the presence of an inflammatory stimulus, such as CXCL1, these effects are then translated into greater levels of firm adhesion and subsequent transmigration. Based on the cited in vitro observations, many of these effects may be $\beta_{2}$ integrin mediated, although the precise adhesive pathways involved in vivo are currently unknown and are under investigation in our laboratory.

As exaggerated leukocyte-vessel wall interactions can lead to leukocyte-mediated vascular damage, a characteristic feature of the pathology of SVV, we investigated the effect of ANCAs on vascular injury as manifested by local microvascular hemorrhage. For this purpose, to investigate ANCA-induced hemorrhage using intravital microscopy, 2 quantification procedures were established as governed by the extent of the responses observed. In both models, evidence was obtained for ANCA-induced microvascular hemorrhage, a response that was largely associated with ANCAinduced leukocyte transmigration. These findings are consistent with in vitro observations, indicating that ANCAs can induce degranulation of primed neutrophils, with consequent release of reactive oxygen species and proteolytic enzymes, responses that under conditions of neutrophil adhesion to endothelium could cause endothelial injury. ${ }^{30}$ Hence, our findings support the general hypothesis that ANCA-induced exaggerated leukocyte-vessel wall interactions can lead to vascular injury. This is in line with the recent description by Xiao et $\mathrm{al}^{13}$ of the induction of SVV in mice by transfer of anti-MPO-rich antibodies.

Collectively, using a novel rat model of EAV, the present results provide the first direct evidence for the ability of ANCAs to induce leukocyte-vessel wall interactions and leukocyte-mediated vascular damage in vivo. We and others have recently defined the role of various treatment modalities for ANCA-associated vasculitis in the clinical arena, such as the duration of cyclophosphamide treatment, ${ }^{31}$ potential roles for anti-TNF biologic therapy, ${ }^{32}$ and the indications for antibody removal by plasma exchange. ${ }^{33}$ We now have the opportunity, using animal models, to design and test therapies targeting the specific pathways activated by ANCAs. This should lead to improved treatment for patients with SVV.

\section{Acknowledgments}

We thank Ms Jennifer Smith, Drs Alan Salama, Efrem Eren, Paul Cook, and Shijun Wang and Prof Patrick Maxwell for their advice.

\section{References}

1. Booth $A D$, Pusey CD, Jayne DR. Renal vasculitis: an update in 2004. Nephrol Dial Transplant. 2004;19:1964-1968.

2. Davies DJ, Moran JE, Niall JF, Ryan GB. Segmental necrotising glomerulonephritis with antineutrophil antibody: possible arbovirus aetiology? Br Med J (Clin Res Ed). 1982;285:606

3. van der Woude FJ, Rasmussen N, Lobatto S, et al. Autoantibodies against neutrophils and monocytes: tool for diagnosis and marker of disease activity in Wegener's granulomatosis. Lancet. 1985;1:425-429.

4. Falk RJ, Jennette JC. Anti-neutrophil cytoplasmic autoantibodies with specificity for myeloperoxidase in patients with systemic vasculitis and idiopathic necrotizing and crescentic glomerulonephritis. N Engl J Med. 1988;318:1651-1657.

5. Falk RJ, Terrell RS, Charles LA, Jennette JC. Anti-neutrophil cytoplasmic autoantibodies induce neutrophils to degranulate and produce oxy- gen radicals in vitro. Proc Natl Acad Sci U S A 1990;87:4115-4119.

6. Williams JM, Ben-Smith A, Hewins P, et al. Activation of the $\mathrm{G}$ (i) heterotrimeric $\mathrm{G}$ protein by ANCA IgG $F\left(a b^{\prime}\right) 2$ fragments is necessary but not sufficient to stimulate the recruitment of those downstream mediators used by intact ANCA IgG. J Am Soc Nephrol. 2003;14:661-669.

7. Weidner S, Neupert W, Goppelt-Struebe M, Rupprecht HD. Antineutrophil cytoplasmic antibodies 
induce human monocytes to produce oxygen radicals in vitro. Arthritis Rheum. 2001;44:16981706.

8. Hewins P, Williams JM, Wakelam MJ, Savage CO. Activation of Syk in neutrophils by antineutrophil cytoplasm antibodies occurs via Fcgamma receptors and CD18. J Am Soc Nephrol. 2004;15: 796-808.

9. Harper L, Cockwell P, Adu D, Savage CO. Neutrophil priming and apoptosis in anti-neutrophil cytoplasmic autoantibody-associated vasculitis. Kidney Int. 2001;59:1729-1738.

10. Radford DJ, Savage CO, Nash GB. Treatment of rolling neutrophils with antineutrophil cytoplasmic antibodies causes conversion to firm integrinmediated adhesion. Arthritis Rheum. 2000;43: 1337-1345.

11. Radford DJ, Luu NT, Hewins P, Nash GB, Savage $\mathrm{CO}$. Antineutrophil cytoplasmic antibodies stabilize adhesion and promote migration of flowing neutrophils on endothelial cells. Arthritis Rheum. 2001;44:2851-2861.

12. Smyth C, Pusey $C$, Haskard DO. ANCA induce leukocyte adhesion to endothelial cells mediated by $E$-selectin and integrins under conditions of flow [abstract]. J Am Soc Nephrol. 2002;13:170A.

13. Xiao $H$, Heeringa $P, H u P$, et al. Antineutrophil cytoplasmic autoantibodies specific for myeloperoxidase cause glomerulonephritis and vasculitis in mice. J Clin Invest. 2002;110:955-963.

14. Smyth C, Smith J, Cook T, Haskard DO, Pusey C Immunisation with MPO directly induces small vessel vasculitis with pauci-immune focal segmental glomerulonephritis and alveolar haemorrhage in WKY rats. J Am Soc Nephrol. 2002;13: $170 \mathrm{~A}$.

15. Thompson RD, Wakelin MW, Larbi KY, et al. Divergent effects of platelet-endothelial cell adhesion molecule-1 and b3 integrin blockade on leukocyte transmigration in vivo. $\mathrm{J}$ Immunol. 2000; 165:426-434
16. Thompson RD, Noble KE, Larbi KY, et al. Platelet-endothelial cell adhesion molecule-1 (PECAM-1)-deficient mice demonstrate a transient and cytokine-specific role for PECAM- 1 in leukocyte migration through the perivascular basement membrane. Blood. 2001;97:18541860.

17. Savige J, Gillis D, Benson E, et al. International consensus statement on testing and reporting of antineutrophil cytoplasmic antibodies (ANCA). Am J Clin Pathol. 1999;111:507-513.

18. Cockwell P, Brooks CJ, Adu D, Savage CO. Interleukin-8: a pathogenetic role in antineutrophil cytoplasmic autoantibody-associated glomerulonephritis. Kidney Int. 1999;55:852-863.

19. Reynolds J, Khan SB, Allen AR, Benjamin CD, Pusey CD. Blockade of the CD154-CD40 costimulatory pathway prevents the development of experimental autoimmune glomerulonephritis. Kidney Int. 2004;66:1444-1452.

20. Brouwer E, Huitema MG, Klok PA, et al. Antimyeloperoxidase-associated proliferative glomerulonephritis: an animal model. J Exp Med. 1993:177: 905-914.

21. Yang JJ, Jennette JC, Falk RJ. Immune complex glomerulonephritis is induced in rats immunized with heterologous myeloperoxidase. Clin Exp Immunol. 1994;97:466-473.

22. Yeung RS, Hino O, Vilensky M, et al. Assignment of 22 loci in the rat by somatic hybrid and linkage analysis. Mamm Genome. 1993;4:585-588

23. Patry $Y C$, Nachman $\mathrm{PH}$, Audrain MA, Falk RJ, Meflah K, Esnault VL. Difference in antigenic determinant profiles between human and rat myeloperoxidase. Clin Exp Immunol. 2003;132:505508.

24. Ewert BH, Jennette JC, Falk RJ. Anti-myeloperoxidase antibodies stimulate neutrophils to damage human endothelial cells. Kidney Int. 1992;41: 375-383.
25. Booth AD, Wallace S, McEniery CM, et al. Inflammation and arterial stiffness in systemic vasculitis: a model of vascular inflammation. Arthritis Rheum. 2004;50:581-588.

26. Johnson PA, Alexander HD, McMillan SA, Maxwell AP. Up-regulation of the granulocyte adhesion molecule Mac-1 by autoantibodies in autoimmune vasculitis. Clin Exp Immunol. 1997;107: 513-519.

27. Nowack R, Schwalbe K, Flores-Suarez LF, Yard $B$, van der Woude FJ. Upregulation of CD14 and CD18 on monocytes In vitro by antineutrophil cytoplasmic autoantibodies. J Am Soc Nephrol. 2000;11:1639-1646.

28. Ewert BH, Becker ME, Jennette JC, Falk RJ. Antimyeloperoxidase antibodies induce neutrophil adherence to cultured human endothelial cells. Ren Fail. 1995;17:125-133.

29. Mayet WJ, Meyer zum Buschenfelde KH. Antibodies to proteinase 3 increase adhesion of neu trophils to human endothelial cells. Clin Exp Immunol. 1993:94:440-446.

30. Savage CO, Gaskin G, Pusey CD, Pearson JD. Anti-neutrophil cytoplasm antibodies can recognize vascular endothelial cell-bound anti-neutrophil cytoplasm antibody-associated autoantigens. Exp Nephrol. 1993;1:190-195.

31. Jayne D, Rasmussen N, Andrassy K, et al. A randomized trial of maintenance therapy for vasculitis associated with antineutrophil cytoplasmic au toantibodies. N Engl J Med. 2003;349:36-44.

32. Booth A, Harper L, Hammad T, et al. Prospective study of TNFalpha blockade with infliximab in anti-neutrophil cytoplasmic antibody-associated systemic vasculitis. J Am Soc Nephrol. 2004;15: 717-721.

33. Gaskin G, Jayne D, Group. EVS: adjunctive plasma exchange is superior to methylprednisolone in acute renal failure due to ANCA-associated glomerulonephritis [abstract]. J Am Soc Nephrol. 2002;13:2A. 